Research Paper

\title{
Variation in chronic nicotinamide treatment after traumatic brain injury can alter components of functional recovery independent of histological damage
}

\author{
Michael R. Hoane, ${ }^{*}$ Jeremy L. Pierce, Nicholas A. Kaufman and Jason E. Beare \\ Restorative Neuroscience Laboratory; Center for Integrative Research in Cognitive and Neural Sciences; Department of Psychology; Southern Illinois University; Carbondale, \\ Illinois USA
}

\begin{abstract}
Abbreviations: TBI, traumatic brain injury; CCI, cortical contusion injury; NAM, nicotinamide; MWM, morris water maze; NA, nicotinic acid; PARP, poly-ADP-ribose polymerase; NAD, nicotinamide adenine dinucleotide; BBB, blood-brain barrier; ROS, reactive oxygen species; ATP, adenosine triphosphate; FPI, fluid percussion injury; ANOVA, analysis of variance; ad lib, ad libitum
\end{abstract}

Key words: traumatic brain injury, therapy, window of opportunity, vitamin, recovery of function

Previously, we have shown that the window of opportunity for nicotinamide (NAM) therapy $(50 \mathrm{mg} / \mathrm{kg}$ ) following cortical contusion injuries (CCI) extended to $4-8 \mathrm{hrs}$ post-CCI when administered over a six day post-CCI interval. The purpose of the present study was to determine if a more chronic NAM treatment protocol administered following CCI would extend the current window of opportunity for effective treatment onset. Groups of rats received either unilateral CCI's or sham procedures. Initiation of NAM therapy $(50 \mathrm{mg} / \mathrm{kg}$, ip) began at either 15-min, 4-hrs, 8-hrs or 24-hrs post-injury. All groups received daily systemic treatments for 12 days post-CCI at $24 \mathrm{hr}$ intervals. Behavioral assessments were conducted for 28 days post injury and included: vibrissae forelimb placing, bilateral tactile adhesive removal, forelimb asymmetry task and locomotor placing testing. Behavioral analysis on both the tactile removal and locomotor placing tests showed that all NAM-treated groups facilitated recovery of function compared to saline treatment. However, on the vibrissae-forelimb placing and forelimb asymmetry tests only the 4-hr and 8-hr NAM-treated groups were significantly different from the saline-treated group. The lesion analysis showed that treatment with NAM out to $8 \mathrm{hrs}$ post-CCI significantly reduced the size of the injury cavity. The window of opportunity for NAM treatment is task-dependent and in some situations can extend to $24 \mathrm{hrs}$ post-CCI. These results suggest that a long term treatment regimen of $50 \mathrm{mg} / \mathrm{kg}$ of NAM starting at the clinically relevant time points may prove efficacious in human TBI.

\footnotetext{
*Correspondence to: Michael R. Hoane; Restorative Neuroscience Laboratory; Department of Psychology; Life Science II; MC 6502; Southern Illinois University; Carbondale, Illinois 62901 USA; Tel.: 618.453.3517; Fax: 618.453.3563; Email: mhoane@siu.edu

Submitted: 06/10/08; Revised: 07/02/08; Accepted: 07/10/08

Previously published online as an Oxidative Medicine and Cellular Longevity E-publication: http://www.landesbioscience.com/journals/oximed/article/6694
}

\section{Introduction}

Each year approximately 50,000 people die from traumatic brain injuries (TBI) and another 80,000 to 90,000 become permanently disabled in the US. ${ }^{1-3}$ Currently, no therapeutics is available to attenuate damage following TBI. A number of novel therapeutics have shown robust behavioral and histological protection in preclinical animal models, but have failed to show beneficial effect in clinical trials. The reason for the lack of translation from animal to human models of TBI is multifaceted, including methodological errors in the clinical setting and errors in preclinical modeling.

Clinically, methodological errors have included patient inclusion criteria and number of patients enrolled to achieve statistical significance. ${ }^{1}$ Some patients may be too severely injured to show any real improvement $(\mathrm{GCS} \leq 8)$; enrolling these patients may artificially deflate any treatment effect. ${ }^{4}$ It has been shown that only 8 of the 203 clinical TBI trials evaluating novel therapeutics were sufficiently powered to detect a $10 \%$ reduction in mortality rate. Thus, some novel therapeutics, given adequate clinical testing, may have been demonstrated to be effective. ${ }^{5}$ Preclinically, neglected aspects of testing novel therapeutics have included: determining the window of opportunity for treatment onset and generalizing treatment to two or more models of cerebral insult. ${ }^{1}$ Treatment duration and dosing parameters should be well defined during the preclinical evaluation of a new therapy. It is unlikely that one or two administrations of a compound will completely arrest its target pathological mechanism and it is possible that acute therapeutic intervention may only delay an inevitable injury cascade; it may take days or weeks of therapeutic intervention to attain efficacy.

Nicotinamide (NAM) is the amide form of niacin (NA or Vitamin B3). NA and NAM are ingested orally in normal dietary intake via yeast, meats, grains and legumes. Although NAM is synthesized endogenously from tryptophan, this reaction does not take place within the central nervous system (CNS) due to the lack of the enzyme quinolinate phosphoribosyl transferase. Thus, any NAM within the CNS has been actively transported across the blood- 
brain barrier (BBB) from the periphery. NAM is a vital component of nicotinamide adenine dinucleotide (NAD), which serves a critical role in aerobic and anaerobic respiration.

NAM elicits a variety of neuroprotective mechanisms post-injury. Following TBI, glutamate chronically excites NMDA and AMPA receptors on neurons. This chronic over-excitation causes a massive influx of calcium $\left(\mathrm{Ca}^{2+}\right)$ into the cells, and begins excitatory amino acid (EAA) induced neurotoxicity. ${ }^{6,7}$ The mitochondria act as a sink for $\mathrm{Ca}^{2+}$, taking up massive amounts. This opens up mitochondrial transition pores, which severely alters ionic homeostasis. High levels of intracellular and mitochondrial $\mathrm{Ca}^{2+}$ trigger a massive release of cytochrome $\mathrm{C}$ which triggers caspase 9 activation leading to terminal apoptosis. NAM activates protein kinase B and inhibits transcription of proapoptotic genes. A downstream effect of protein kinase B is closing of the mitochondrial transition pores opened by excessive $\mathrm{Ca}^{2+}$ influx. The mitochondrion is able to regain ionic homeostasis and bring cytochrome $\mathrm{C}$ back to physiological conditions thereby inhibiting caspase 9 mediated cell death. ${ }^{8}$

NAM also exerts protective effects by inhibiting poly-ADPribose polymerase (PARP). Due to oxidative damage caused by the failure of the electron transport chain and propagation of reactive oxygen species (ROS), DNA becomes damaged.' Following breaks in DNA strands caused by oxidative damage, PARP binds to single or double strand breaks and cleaves NAD to catalyze the transfer of ADP-ribose, which binds to acceptor proteins and to PARP itself reducing amount of free NAD for normal energy metabolism. ${ }^{10}$ Chronic PARP activation quickly depletes cellular NAD stores; administration of NAM has been shown to inhibit PARP, increase levels of NAD in cortical areas affected by ischemic events, and restore ATP levels. ${ }^{11-13}$

Recent research has shown that while NAM administration increases brain concentrations of NAD, the effect of this increase may only serve to inhibit PARP and decrease oxidative stress and not restore metabolic functioning. ${ }^{11}$ Peak plasma concentrations of NAM have been demonstrated within $45 \mathrm{~min}$ after systemic injection in the rat. ${ }^{14}$ The increase of cortical NAD and how long this elevation might last after a $50 \mathrm{mg} / \mathrm{kg}$ dose of NAM is not known. However, if this effect is short lived after a single bolus injection, then treatment beginning at $8 \mathrm{hrs}$ may not have spiked NAD levels at a therapeutically relevant time or delayed pathological mechanisms. It has been reported that PARP demonstrates two peaks following injury, one at $30 \mathrm{~min}$ and at $24 \mathrm{hrs} .{ }^{15}$ It is possible that single administrations of NAM at later time points (i.e., 6-8 hrs) may temporarily attenuate pathological mechanisms upstream of PARP activation, thus pushing the second peak of PARP somewhere outside of the treatment range. In contrast, if the effects of PARP activation were apparent at $24 \mathrm{hrs}$, spiking the concentration of NAD at this time would have produced the therapeutic effect observed following treatment onset at 24 hrs. Thus, the initial protection observed would be a result of acute prevention of apoptosis and not mainly due to restoration of metabolic function. Therefore, it is of interest to determine the effect of repeated dosing of NAM for the treatment of TBI because by increasing the duration of therapy it is likely to increase the beneficial effects on recovery of function.

NAM was first investigated in experimental models of ischemia. Administration of NAM following permanent middle cerebral artery occlusion was effective at reducing infarction volumes in male rats. ${ }^{16}$
This study began treatment $1 \mathrm{hr}$ prior to ischemia onset and tested the effectiveness of three doses: $50 \mathrm{mg} / \mathrm{kg}, 500 \mathrm{mg} / \mathrm{kg}$ and $1000 \mathrm{mg} /$ $\mathrm{kg}$. Histological data revealed an inverted U-shaped distribution with the 50 and $1000 \mathrm{mg} / \mathrm{kg}$ groups failing to show neuroprotection as assessed by infarction volume. The $500 \mathrm{mg} / \mathrm{kg}$ group showed a significant reduction in infarction volume relative to saline $24 \mathrm{hrs}$ post-stroke. A single dose of $500 \mathrm{mg} / \mathrm{kg}$ of NAM was found to have a window of opportunity that extended to $2 \mathrm{hrs}$, but not 3 or 4 hrs post-stroke using a measure of infarction volume. ${ }^{16}$ In another study, a $500 \mathrm{mg} / \mathrm{kg}$ dose was the most effective at reducing infarction volume; the window of opportunity remained at 2 hrs. ${ }^{17}$ It was later demonstrated that the window of opportunity extended to $4 \mathrm{hrs}$ post-stroke with the $500 \mathrm{mg} / \mathrm{kg}$ dose as measured by infarction and neuroscore at 7 days post-stroke. ${ }^{18}$

Recently, NAM has been demonstrated to be effective in models of TBI. It has been shown that NAM was effective at improving behavioral and histological outcome following experimental TBI. ${ }^{19}$ Animals received a bilateral CCI to the medial prefrontal cortex and were administered NAM $(500 \mathrm{mg} / \mathrm{kg})$ at $15 \mathrm{~min}$ with a booster at 24 hrs post injury. Behavioral evaluation revealed that NAM-treated animals were significantly less impaired than saline-treated animals on sensorimotor (e.g., and cognitive measures) but not on a skilled forelimb use task. NAM was also shown to decrease the size of the lesion cavity and downregulate the glial response. ${ }^{19}$ It has also been recently shown that NAM administration acutely reduced apoptosis, BBB breach and neuronal death following CCI. ${ }^{20,21}$

NAM was also effective in attenuating behavioral and histological measures at two different doses in a diffuse model of TBI: fluid percussion injury (FPI). ${ }^{22}$ Animals received FPI injuries and were treated with either $500 \mathrm{mg} / \mathrm{kg}$ or $50 \mathrm{mg} / \mathrm{kg}$ NAM at $15 \mathrm{mins}$ post injury with a single $24 \mathrm{hr}$ booster. Behavioral evaluation over 35 testing days showed significant performance improvement relative to saline-treated animals on measures of sensorimotor functioning, sensorimotor integration, and motor functioning. The $500 \mathrm{mg} /$ $\mathrm{kg}$ treatment condition was also effective in attenuating cognitive dysfunction, but the $50 \mathrm{mg} / \mathrm{kg}$ was not. Histologically, both treatment conditions significantly reduced cavity size and glial cell proliferation relative to saline-treated animals. ${ }^{22}$ Acute neuroprotection and a time-dependent modulation of reactive gliosis at $24 \mathrm{hrs}$ and 7 days post-FPI has also been recently shown. ${ }^{23}$

A recent study has evaluated the window of opportunity for treatment onset using a $50 \mathrm{mg} / \mathrm{kg}$ dose following CCI. Animals received injection regimens starting at 15 -min, 4-hr or 8-hr post injury with $50 \mathrm{mg} / \mathrm{kg}$ boosters at $24 \mathrm{hr}$ intervals for five days following bilateral frontal CCI. Testing revealed that the 15 -min, 4-hr and 8 -hr treatment groups were significantly less impaired in the sensorimotor and sensory tasks. However, only the 15-min and 4-hr treatment groups demonstrated a significant reduction in cognitive deficits. Histologically, the 15-min and 4-hr treatment groups had significantly smaller cavities than saline-treated animals. ${ }^{24}$

The objective of the present study was to extend the window of opportunity of NAM administration from 4 hrs to 24 hrs post injury. Animals were administered NAM for 12 days using the clinically relevant dose of $50 \mathrm{mg} / \mathrm{kg}$. Administration regimens overlapped the duration of time that metabolic dysfunction has been proposed to occur. $^{25,26}$ 


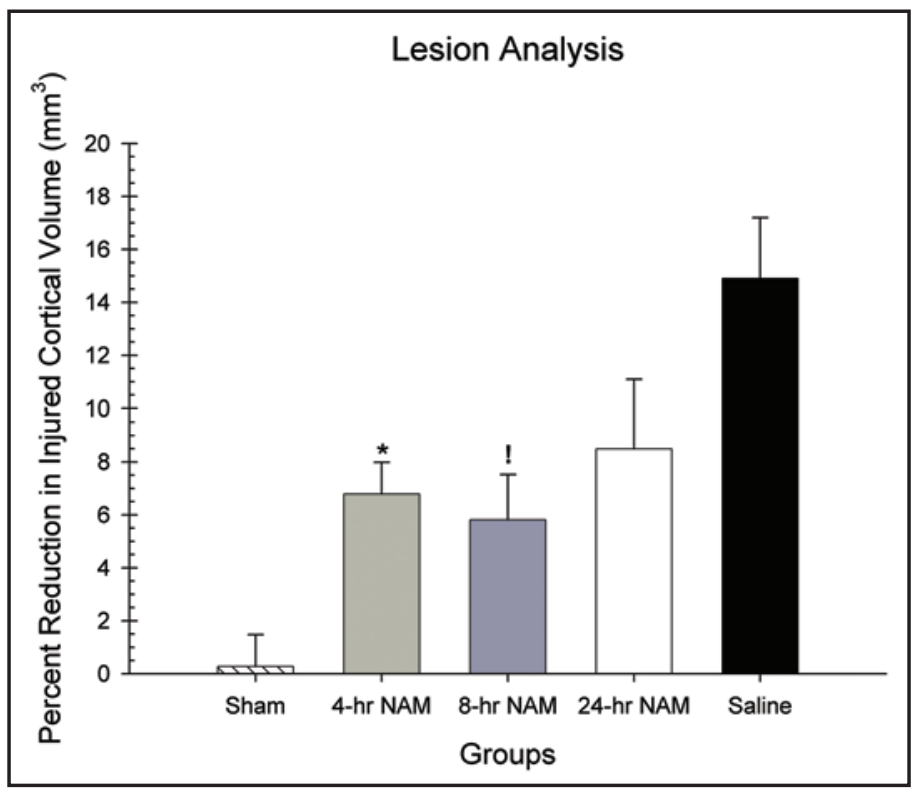

Figure 1. Lesion analysis. Plotted is the mean $( \pm S E M)$ remaining cortical volume for each group. NAM at the $4-h r\left({ }^{*} p<0.05\right)$ and 8 -hr $(! p<0.05)$ time points significantly reduced the amount of injury-induced tissue loss compared to the vehicle group.

\section{Results}

Lesion analysis. Examination of the extent of injury measured by the percent reduction of lesion volume in the ipsilateral hemisphere compared to the intact contralateral hemisphere was analyzed in a one way ANOVA ( $4 \mathrm{hr}-\mathrm{NAM} 8 \mathrm{hr}-\mathrm{NAM}, 24 \mathrm{hr}-\mathrm{NAM}$, Saline and Sham). A significant group effect was observed, $[F(4,32)=6.70$, $\mathrm{p}<0.001]$. Post hoc analysis with Tukey's HSD demonstrated the $4 \mathrm{hr}-\mathrm{NAM}[\operatorname{HSD}(13)=8.10, \mathrm{p}<0.05]$ and $8 \mathrm{hr}-\mathrm{NAM}$ groups $[\operatorname{HSD}(14)=9.06, \mathrm{p}<0.02]$ were significantly different from the saline-treated groups but the $24 \mathrm{hr}-\mathrm{NAM}$ group was not $(\mathrm{p}>0.10)$ (see Fig. 1). However, shams were not significantly different from any of the NAM-treated groups in respect to cortical volume reduction ( $p>0.05$ ). Representative histological images through the site of injury are presented in Figure 2.

Bilateral tactile adhesive removal task. The latency to remove the adhesive patch from the right forelimb was examined using a $5 \times 8$ ANOVA with repeated measures. Group ( 4 hr-NAM, 8 hr-NAM, 24 hr-NAM, Saline and Sham) and day (2, 4, 6, 8, 10, 12, 21 and 28 post-CCI) were included as the between and within group factors, respectively. All animals showed improved latencies to remove the adhesive patch across testing days, the main effect for day was significant $[F(7,224)=33.16, p<0.001]$. A significant group main effect was observed in the latency to remove the adhesive patches from affected forelimb $[F(4,32)=11.04, p<0.001]$. The group $x$ day interaction was also significant, suggesting differential recovery between groups across time $[\mathrm{F}(28,224)=3.00, \mathrm{p}<0.001]$ (See Fig. $3)$. Post hoc comparisons with planned t-tests revealed that the 4 hr-NAM group was significantly different from the saline-treated group on days $2[\mathrm{t}(13)=3.50, \mathrm{p}<0.008], 4[\mathrm{t}(13)=3.82, \mathrm{p}<0.004]$ and $6[t(13)=2.47, \mathrm{p}<0.04]$. Comparisons of the $8 \mathrm{hr}-\mathrm{NAM}$ group to saline were also significantly different on days $4[\mathrm{t}(14)=2.21, \mathrm{p}<$

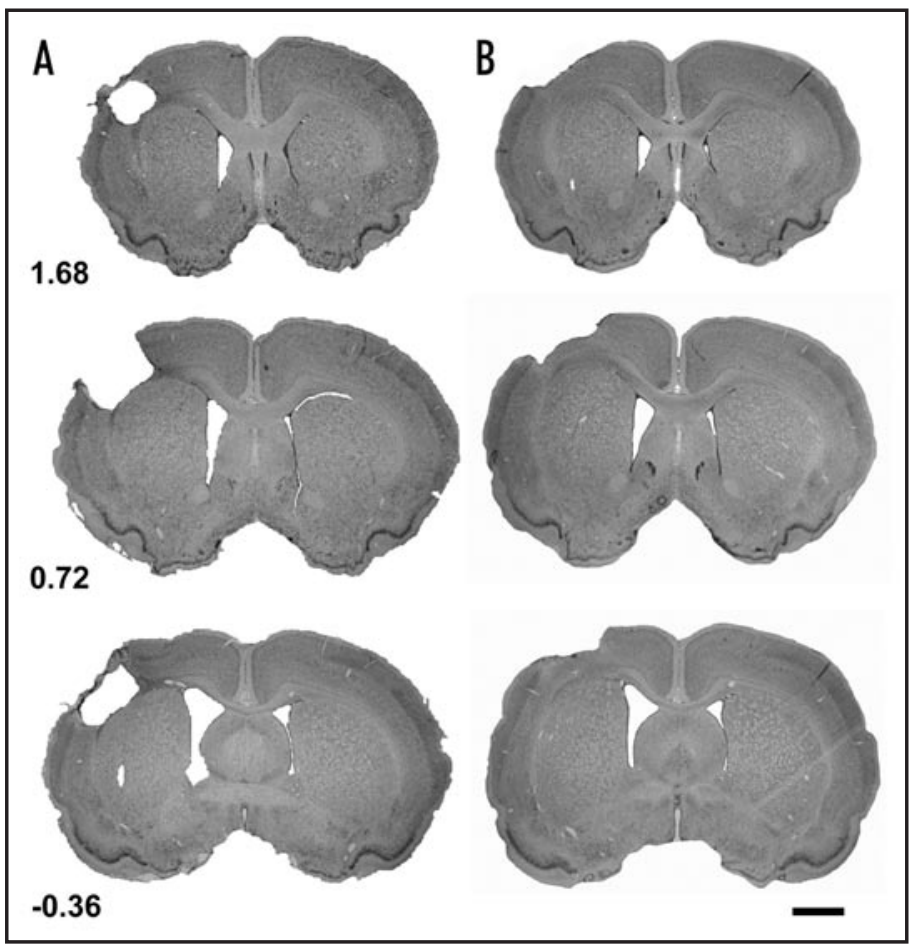

Figure 2. Histology plate. Shown are representative cresyl violet images $(40 \mu \mathrm{m})$ of sections from the saline-treated injured brain $(A)$ and an 8-hr NAM brain (B) at coordinates $1.68 \mathrm{~mm}, 0.72 \mathrm{~mm}$ and $-0.36 \mathrm{~mm}$ relative to bregma $(0.44 x$, scale bar $=2 \mathrm{~mm})$.

$0.05], 6[t(14)=3.63, p<0.003]$ and $8[t(14)=2.17, p<0.05]$. The comparisons between the $24 \mathrm{hr}-\mathrm{NAM}$ and saline-treated group were significantly different on days $2[\mathrm{t}(14)=2.59, \mathrm{p}<0.02], 4[\mathrm{t}(14)=$ $4.09, \mathrm{p}<0.002]$ and $6[\mathrm{t}(14)=3.20, \mathrm{p}<0.008]$, and $8[\mathrm{t}(14)=2.87$, $\mathrm{p}<0.02]$. Comparisons between the NAM-treated groups were not significantly different $(\mathrm{p}>0.05)$.

Vibrissae-forelimb placing. The percentage of unsuccessful placing attempts with the contralateral forelimb was examined using a 5 x 9 ANOVA with repeated measures. Group ( 4 hr-NAM, 8 hr-NAM, 24 hr-NAM, Saline and Sham) and day $(2,4,6,8,10$, $12,14,21$ and 28 post-CCI) were included as the between and within group factors, respectively. There was a general improvement in recovery of function across testing days, the main effect for day was significant $[F(8,256)=16.89, p<0.001]$. A significant group main effect was observed in placing performance in the affected contralateral forelimb $[\mathrm{F}(4,32)=12.34, \mathrm{p}<0.001]$. The group $\mathrm{x}$ day interaction was also significant, suggesting differential improvement between groups $[\mathrm{F}(32,256)=2.59, \mathrm{p}<0.003]$ (See Fig. 4). Post hoc comparisons with planned t-tests revealed that the $4 \mathrm{hr}$-NAM group was significantly different from the saline-treated group on days 12 $[\mathrm{t}(13)=2.85, \mathrm{p}<0.01], 14[\mathrm{t}(13)=3.79, \mathrm{p}<0.002], 21[\mathrm{t}(13)=$ $3.69, \mathrm{p}<0.003]$ and $28[\mathrm{t}(13)=3.45, \mathrm{p}<0.004]$. Comparisons of the $8 \mathrm{hr}$-NAM group to saline were also significantly different on days $8[\mathrm{t}(14)=2.55, \mathrm{p}<0.02], 10[\mathrm{t}(14)=2.65, \mathrm{p}<0.02], 12[\mathrm{t}(14)$ $=2.60, \mathrm{p}<0.02], 14[\mathrm{t}(14)=2.67, \mathrm{p}<0.02], 21[\mathrm{t}(14)=2.88, \mathrm{p}<$ $0.01]$ and $28[\mathrm{t}(14)=2.68, \mathrm{p}<0.02]$. The comparisons between the $24 \mathrm{hr}-\mathrm{NAM}$ and saline-treated group were not significantly different $(p>0.05)$. Comparisons between the NAM-treated groups were not significantly different $(\mathrm{p}>0.05)$. 


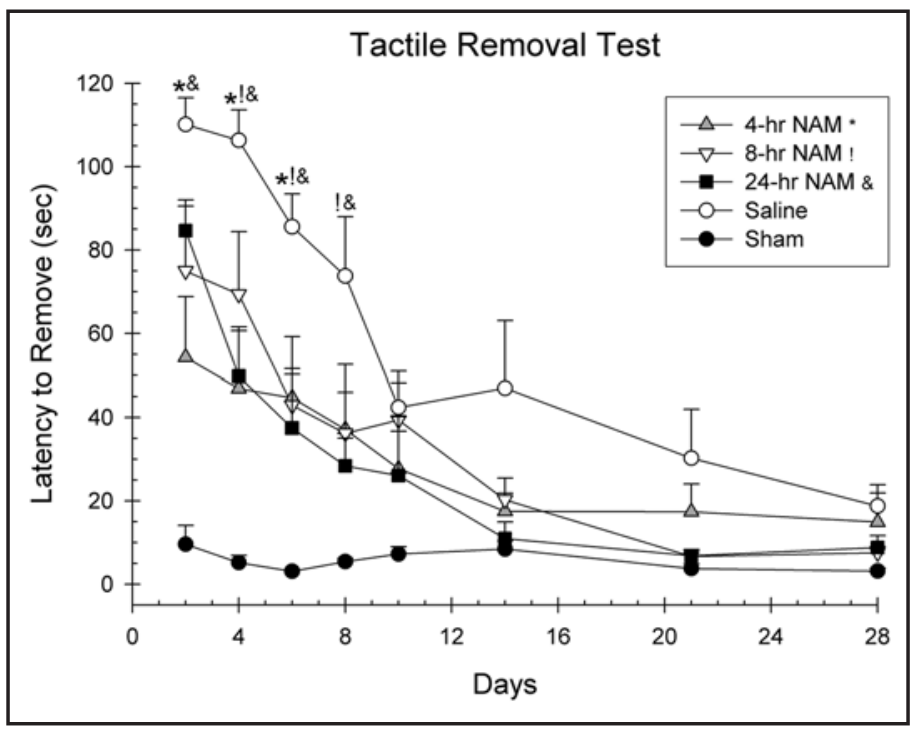

Figure 3. The effects of a 13 day regimen of NAM $(50 \mathrm{mg} / \mathrm{kg}$, ip) or saline administered following $\mathrm{CCl}$ or sham surgery on the bilateral tactile adhesive removal test. The graph shows the plotted mean $( \pm S E M)$ latencies to remove the stimuli from both forelimbs. Treatment with the 4-hr, 8-hr and 24-hr NAM groups significantly improved performance compared to the saline-treated group. Symbols indicate significant differences $(p<0.05)$ between comparisons of NAM-treated groups and the saline-treated group.

Locomotor placing. The impairment of the forelimbs during horizontal locomotion on a grid floor was examined using a $5 \times 3$ ANOVA with repeated measures. Group ( 4 hr-NAM, 8 hr-NAM, $24 \mathrm{hr}-\mathrm{NAM}$, Saline and Sham) and day (2, 8 and 14 post-CCI) were included as the between and within group factors, respectively. Improved forelimb performance was generally observed across days, the main effect for day was significant $[F(2,64)=24.82, p<0.001]$. A significant group main effect was observed in horizontal forelimb performance $[F(4,32)=30.84, p<0.001]$. The group $x$ day interaction was also significant, suggesting differential recovery between groups across time $[\mathrm{F}(8,64)=3.00, \mathrm{p}<0.001]$ (See Fig. 5). Post hoc comparisons with planned t-tests revealed that the $4 \mathrm{hr}-\mathrm{NAM}$ group was significantly different from the saline-treated group on days 2 $[\mathrm{t}(13)=19.67, \mathrm{p}<0.001], 8[\mathrm{t}(13)=7.57, \mathrm{p}<0.001]$ and $14[\mathrm{t}(13)$ $=8.99, \mathrm{p}<0.001]$. Comparisons of the $8 \mathrm{hr}-\mathrm{NAM}$ group to saline were also significantly different on days $2[\mathrm{t}(14)=3.75, \mathrm{p}<0.002]$, $8[\mathrm{t}(14)=3.55, \mathrm{p}<0.003]$ and $14[\mathrm{t}(14)=2.87, \mathrm{p}<0.01]$. The comparisons between the $24 \mathrm{hr}-\mathrm{NAM}$ and saline-treated group were significantly different on days $2[\mathrm{t}(14)=2.97, \mathrm{p}<0.01]$ and $8[\mathrm{t}(14)$ $=2.75, \mathrm{p}<0.02]$. Comparisons between the NAM-treated groups showed that the $4 \mathrm{hr}$-NAM group was significantly different than the $8 \mathrm{hr}-\mathrm{NAM}$ and $24 \mathrm{hr}-\mathrm{NAM}$ groups at all 3 test days $(\mathrm{p}<0.05)$.

Forelimb asymmetry. Forelimb asymmetry use during rearing behavior was examined using a $5 \times 3$ ANOVA with repeated measures. Group ( 4 hr-NAM, 8 hr-NAM, 24 hr-NAM, Saline and Sham) and day (2, 6 and 12 post-CCI) were included as the between and within group factors, respectively. Improved forelimb asymmetry was generally observed across days, the main effect for day was significant $[\mathrm{F}(2,64)=15.52, \mathrm{p}<0.001]$. A significant group main effect was observed in forelimb asymmetry $[\mathrm{F}(4,32)=14.50, \mathrm{p}<0.001]$. The group $\mathrm{x}$ day interaction was not significant, suggesting a lack of

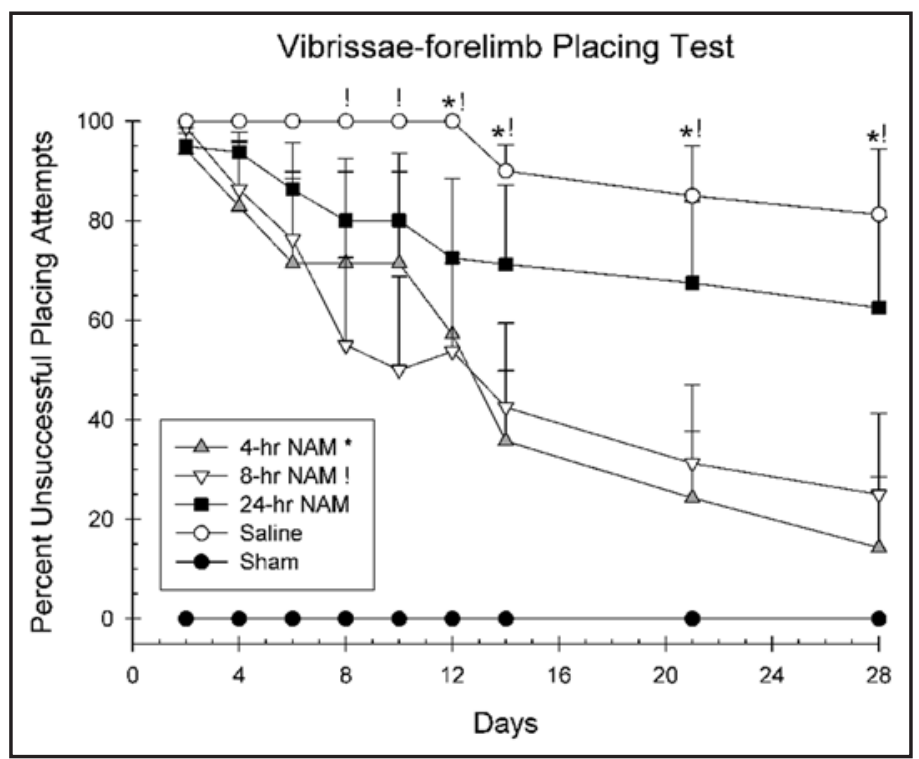

Figure 4. The effects of a 13 day regimen of NAM or vehicle administered following frontal $\mathrm{CCl}$ or sham surgery on the vibrissae-forelimb placing test. The graph shows the plotted mean $( \pm S E M)$ percentage of unsuccessful placing attempts. Treatment with the 4 -hr and 8 -hr NAM groups significantly improved performance compared to the saline-treated group. Symbols indicate significant differences $(p<0.05)$ between comparisons of NAM-treated groups and the saline-treated group.

differential recovery between groups across time $[F(8,64)=0.92, p>$ $0.50]$. With the non-significant interaction effect, post hoc comparisons with planned Tukey HSD tests were performed between groups. This comparison revealed that the $4 \mathrm{hr}-\mathrm{NAM}$ group was significantly different from the saline-treated group $[\operatorname{HSD}(13)=19.67, \mathrm{p}<$ 0.001] (See Fig. 6). Comparisons of the $8 \mathrm{hr}-\mathrm{NAM}$ group to saline were also significantly different $[\operatorname{HSD}(14)=3.75, \mathrm{p}<0.002]$. The $24 \mathrm{hr}-\mathrm{NAM}$ group was not significantly different compared to the saline group $[\operatorname{HSD}(14)=3.75, \mathrm{p}<0.002]$. Comparisons between the $4 \mathrm{hr}$-NAM group were only significantly different from the 24 hr-NAM on the first test day $(\mathrm{p}<0.05)$.

\section{Discussion}

This study sought to extend the window of opportunity for NAM therapy by prolonging administration to include time points covering metabolic dysfunction post-TBI. Animals within the present study received NAM at the clinically relevant dose of $50 \mathrm{mg} / \mathrm{kg}$ and at clinically relevant administration times; 4, 8 and 24 hrs post-injury with 12 days of daily boosters. The injury model utilized produced enduring deficits in forelimb functioning as assessed in locomotor placing, forelimb asymmetry, bilateral tactile adhesive removal, and vibrissae forelimb placing over the course of 28 days. Histological evaluation was performed to evaluate the treatment regimen's ability to reduce injury extent.

The results of this study have shown that administration of NAM following CCI had a task dependent effect on the window of opportunity of recovery of function. In general, the 13-dose regimen of NAM significantly lessened the behavioral impairments observed following injury and led to a more rapid and sustained improvement in functional recovery. On 2 of the 4 behavioral tests examined the window of opportunity for this dosing regimen of NAM extended 


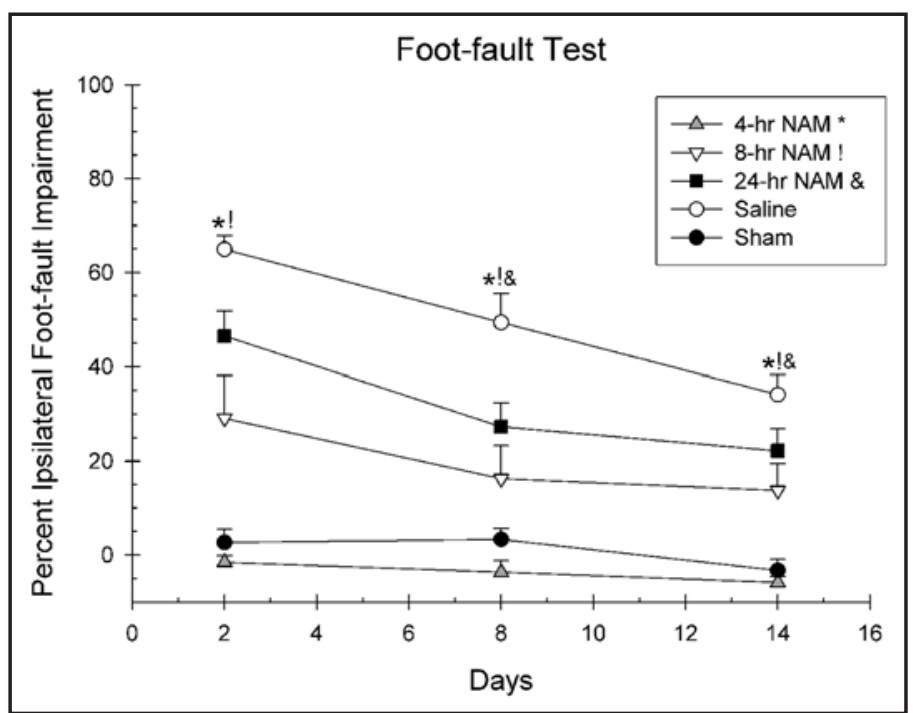

Figure 5. The effects of a 13 day regimen of NAM $(50 \mathrm{mg} / \mathrm{kg}$, ip) or saline administered following $\mathrm{CCl}$ or sham surgery on the locomotor placing test. The graph shows the plotted mean $( \pm$ SEM) forelimb foot-faults impairment scores. Treatment with the 4-hr, 8-hr and 24-hr NAM groups significantly improved performance compared to the saline-treated group. Symbols indicate significant differences $(p<0.05)$ between comparisons of NAM-treated groups and the saline-treated group.

out to $24 \mathrm{hrs}$ post-CCI. On the bilateral tactile removal test it was found that administration of NAM at the 4-hr, 8-hr and 24-hr postCCI time points significantly improved recovery of function on this test. On post-CCI day 2 it was found that both the 4-hr and 8-hr groups showed a significantly reduced initial impairment on this test; and the 24-hr group showed a greatly reduced impairment, compared to the saline-treated group. Over the first week of testing all of the NAM groups showed a significantly improved level of performance compared to the saline-treated group. In general, the data from the bilateral tactile removal test demonstrates that there were no significant differences between any of the NAM treatment groups. Thus, on this test a very wide window of opportunity was present. A similar level of performance was also shown on the locomotor placing test. The 4-hr NAM group demonstrated no behavioral impairments on this test for the extent of the testing period post-CCI. The 8-hr NAM group was significantly reduced on all test days compared to the saline-treated group and the 24-hr group showed a strong reduction on the first test day and significant reductions on all subsequent test days. Comparisons between the NAM-treated groups showed that the 4-hr group was significantly improved compared to the 8 and 24-hr groups. Inspection of the graph in figure 5 reveals a classic window of opportunity effect for NAM therapy post-CCI, with the earlier dosing points providing superior behavioral performance compared to later time points; however, in this case all of the NAM treatment groups significantly outperformed the vehicle control group. Thus, the earlier the treatment can be initiated post-injury the better the expected recovery outcome. In general, on these 2 behavioral tests (bilateral tactile removal and locomotor placing) the window of opportunity extends out to $24 \mathrm{hrs}$ post-CCI.

The results of the vibrissae-forelimb placing test showed that all of the NAM-treated animals showed some degree of recovery compared to the saline-treated animals, with most of the significant recovery

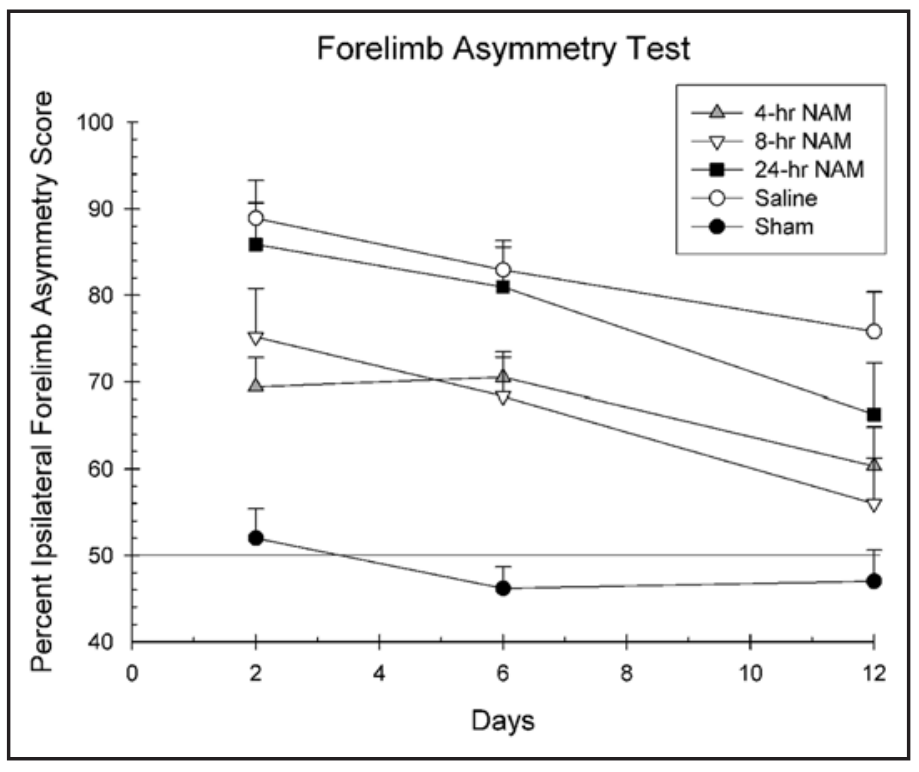

Figure 6. The effects of a 13 day regimen of NAM or vehicle administered following frontal $\mathrm{CCl}$ or sham surgery on the forelimb asymmetry test. The graph shows the plotted mean $( \pm S E M)$ percentage of forelimb asymmetry scores. The no bias line at 50\% demonstrates a level of performance where both forelimbs are being used equally on this test. Treatment with the 4-hr and 8-hr NAM groups significantly improved performance compared to the saline-treated group. Symbols indicate significant differences $(p<0.05)$ between comparisons of NAM-treated groups and the saline-treated group.

occurring after 10 days of testing which is typical for this particular behavioral test. However, on this test only the 4-hr and 8-hr NAM groups were significantly improved compared to saline treatment. Treatment with NAM at the 4-hr or 8-hr time points resulted in approximately an $80-90 \%$ improvement in placing performance compared to the saline-treated group which showed only a $20 \%$ improvement in performance by the end of testing. The 24-hr NAM group failed to produce significant improvement in performance on this test; however, a limited degree of improvement was observed compared to the saline-treated group. The recovery curves on the vibrissae-forelimb placing tests correlate very nicely with the tissue sparing data and may suggest that this behavioral test may be the most sensitive for detecting anatomical sparing. In a similar manner, the analysis of the forelimb asymmetry test demonstrated the same general finding. Treatment with NAM starting at either 4-hr or 8-hr significantly reduced the forelimb asymmetry bias observed after injury compared to saline treatment. There was little improvement in the 24-hr NAM group compared to the saline-treated group until the last day of testing. It is unknown if this improvement was due to an effect of NAM treatment, or the result of spontaneous recovery of function (as occurred in the saline-treated group); however, the improvement in the saline-treated group between days 6 and 12 were non-significant. Thus, on these 2 sensorimotor tests NAM administered as late as $8 \mathrm{hrs}$ post-CCI significantly improved recovery of function; whereas, there were only slight improvements when NAM was administered at $24 \mathrm{hrs}$ post-CCI. In general, the behavioral window of opportunity following CCI can be extended out to 24 hrs post-injury with sustained daily dosing of low dose NAM therapy; however, this behavioral improvement appears to be task dependent. A very consistent effect was observed when NAM 
therapy was initiated at $8 \mathrm{hrs}$ post-CCI which clearly represents a clinically relevant treatment window.

The results of the anatomical analysis also showed a time-dependent effect for NAM treatment. Both the 4-hr and 8-hr treatment groups showed a preservation of cortical tissue loss following CCI compared to the saline-treated group. The 24-hr group was not significantly different compared to the saline-treated group; however, the cortical loss was reduced. In a previous window of opportunity study for NAM therapy it was found that 6 daily doses of low dose NAM did not provide significant cortical protection following CCI. ${ }^{24}$ Thus, it appears that by increasing the length of NAM therapy post-CCI that greater beneficial effects can be observed. This finding suggests that by providing NAM therapy during the time of metabolic crisis following CCI that a significant gain in tissue sparing and behavioral recovery can be achieved.

The results of this study indicate that a 13-dose regimen of NAM significantly improved performance on all behavioral measures of sensorimotor function when administered as late as $8 \mathrm{hrs}$ post-CCI. Furthermore, significant improvements in sensorimotor performance were observed when administered as late as $24 \mathrm{hrs}$ post-CCI on certain tests. NAM administration also significantly reduced tissue loss in the injured cortex when administered 8 hrs following injury. In conclusion, by increasing the length of time of treatment duration the window of opportunity can be extended for NAM therapy in the traumatically injured rodent brain.

\section{Methods}

Subjects. Forty male Sprague-Dawley rats, 3-5 months of age were included in this experiment. All experimental procedures were reviewed and approved by the Institutional Animal Care and Use Committee. The study was conducted in a facility certified by the American Association for the Accreditation of Laboratory Animal Care. Rats were maintained on a standard 12-hr light/dark cycle with food and water available ad lib.

Surgery. The surgical procedure was performed using aseptic techniques and conditions. The CCI model utilized in the proposed study is based on previous studies. ${ }^{19,27}$ Animals were anesthetized using a mixture of isoflurane $(2-4 \%)$ and oxygen $(0.8 \mathrm{~L} / \mathrm{min})$. When the animal became unresponsive (no ocular or pedal reflexes) the head was shaved and scrubbed with 70\% alcohol and placed into a stereotaxic device. A midline incision was made in the skin and underlying fascia was reflected. A circular craniotomy $(4.0 \mathrm{~mm})$ was performed using a surgical drill. Care was taken to avoid damaging the meninges and cortex while drilling. The craniotomy was performed $0.5 \mathrm{~mm}$ anterior to bregma and $-4.0 \mathrm{~mm}$ lateral to bregma exposing the cortical region containing sensorimotor forelimb representation. The contusion injury was created with a sterile, stainless steel impactor tip (3.0 $\mathrm{mm}$ in diameter) that was attached to a magnetically activated piston (www.myneurolab.com) set at an angle of $10^{\circ}$ to insure that the surface of the impact tip and cortex were parallel at the time of impact. The impact tip traveled at a velocity of $2.5 \mathrm{~m} / \mathrm{s}$ compressing the cortex to a depth of $2.0 \mathrm{~mm}$ during $0.5 \mathrm{sec}$ of contact. Following contusion any bleeding was controlled with sterile sponges soaked in cold saline and the incision was closed with nylon suture material. To maintain normal body temperature during surgery and recovery, the rats were maintained on normothermic $\left(37^{\circ} \mathrm{C}\right)$ heating units $(\mathrm{EZ}$ Anesthesia, Inc.,). Rats receiving sham surgeries underwent identical

\section{Table 1 Experimental Design}

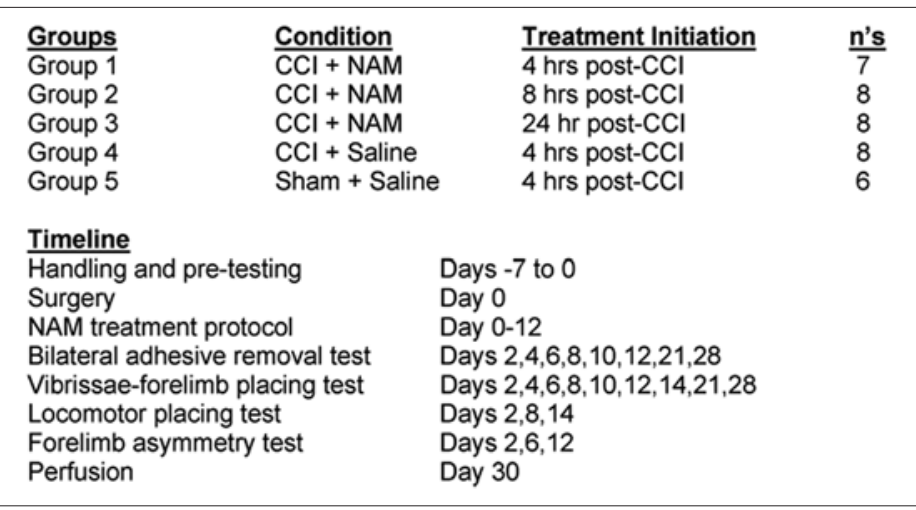

surgical prep as injured animals, received craniotomies, and then were sutured.

Drug administration. Following injury NAM-treated animals received NAM injections $(50 \mathrm{mg} / \mathrm{kg}$, i.p.) (Sigma \# N3376, St. Louis, MO, USA $)$ at 4 -hr $(\mathrm{n}=8), 8-\mathrm{hr}(\mathrm{n}=8)$ or $24-\mathrm{hr}(\mathrm{n}=8)$ post-injury, followed by daily booster $(50 \mathrm{mg} / \mathrm{kg}$, i.p.) injections at $24 \mathrm{hr}$ intervals for 12 days. $^{22}$ Sham $(\mathrm{n}=8)$ and injured-control $(\mathrm{n}=8)$ animals received saline injections $(0.9 \%, 1.0 \mathrm{~mL} / \mathrm{kg}$, i.p. $) 4$ hr post-injury followed by 12 daily $(0.9 \%, 1.0 \mathrm{~mL} / \mathrm{kg}$, i.p.) boosters at $24 \mathrm{hr}$ intervals (see Table 1 for assignments). All behavioral testing and histological analysis were carried out by experimenters blinded to treatment conditions.

Bilateral tactile adhesive removal test. This procedure has been previously shown to be a sensitive assessment of somatosensory and attentional deficits following injury to the sensorimotor cortex and has been described in detail. ${ }^{19,28-30}$ A slightly modified version of this task was used in this experiment. A small rectangular patch (105 $\mathrm{mm}^{2}$ ) (Avery, product \#05412) was applied to the radial aspect of each forelimb. The rat was returned to the home cage and the latency and order of contact and removal (right vs. left) of the stimuli was recorded. A trial ended when the rat either removed both patches or 2 minutes had elapsed. Baseline latencies were recorded prior to CCI after which animals were tested on two trials per testing day (ITI 5 $\min$ ), on post-operative days $2,4,6,8,10,12,21$ and 28. Refer to Table 1 for complete testing schedules.

Vibrissae-forelimb placing. Sensorimotor function was evaluated by scoring forelimb placing reaction. ${ }^{22,24,30}$ Each rat was held by the trunk ensuring the forelimbs were free to move. One side of the rat was oriented parallel to a Plexiglas surface and was slowly moved until the vibrissae on one side touched the surface. A reliable lateralized placing response was elicited in intact rats each time the vibrissae made contact with the surface. A successful forelimb placing response was recorded if the animal raised its forelimb and placed it on the surface in response to stimulation of the vibrissae ipsilateral to the forelimb. Each rat was given 10 trials for each forelimb. If a placing response was not elicited within $5 \mathrm{sec}$ of vibrissae stimulation, the trial was recorded as unsuccessful. Baseline performance was recorded prior to injury. The animals were tested on post-operative days 2, 4, $6,8,10,12,14,21$ and 28.

Locomotor placing. This test assessed the recovery of coordinated limb placing during locomotion. ${ }^{30,31}$ Each rat was placed 
on an elevated grid floor $(56.5 \times 54 \mathrm{~cm})$ and allowed to explore for 3 min while being videotaped. The openings in the grid were 3.5 $\mathrm{cm}^{2}$. While moving around the grid an intact animal occasionally made a "foot-fault"; this occurred when a rat inaccurately placed a limb and it fell through a hole in the grid floor. Over the course of testing, intact animals made few foot-faults. A rat with a lesion to the sensorimotor cortex will make foot-faults with the forelimb contralateral to the injury. To weight the frequency of foot-faults relative to amount of movement the number of steps was recorded. The deficit assessment was calculated using the equation:

\section{[((contralateral fault - ipsilateral fault)/\# steps) x 100].}

The animals were tested on post-operative days 2, 8 and 14 .

Forelimb asymmetry task. Following unilateral injury to the sensorimotor cortex animals display a preference for the unimpaired forelimb. ${ }^{24,30}$ To assess this bias, animals were placed in a $20 \times 20$ x $30 \mathrm{~cm}$ clear cage and videotaped for $5 \mathrm{~min}$ while exploring the walls of the cage. During rearing behavior, intact animals used both forelimbs equally during vertical and horizontal movements. An impaired animal reared and supported its weight against the wall with the unaffected forelimb and during horizontal movements. While scoring the taped session a movement in which both forelimbs made contact with the wall of the cage either at the same time or in short succession was scored as a "both". Rears in which the animal made either a left or right placement singly was scored as left or right. Weight bearing movements were considered those forelimb places in which the palm of the forepaw made contact with the glass and/ or remained stable until the animal moved horizontally or vertically. A score of forelimb asymmetry bias was calculated by the following equation:

$[($ ipsilateral $+1 / 2$ both $) /($ ipsilateral + contralateral + both $) \times 100]$.

The animals were tested on post-operative days 2, 6 and 12 .

Histology. At 30 days post-injury, rats were anesthetized with urethane $(3.0 \mathrm{~g} / \mathrm{kg}, 0.5 \mathrm{~g} / \mathrm{ml}$, i.p. $)$ and transcardially perfused with $0.9 \%$ phosphate buffered saline (PBS) followed by $10 \%$ phosphate buffered formalin (PBF). Brains were removed from the cranium and post-fixed in PBF. Brains were then placed in a $30 \%$ sucrose solution for 3 days for cryprotection prior to being sectioned. Brains were serially sectioned at $40 \mu \mathrm{m}$ on a sliding microtome with an electronic freezing stage and stored in phosphate buffered saline.

Lesion analysis. A series of slides were stained with cresyl violet, dehydrated and cover slipped. The extent of the lesion was captured with an Olympus microscope (BX-51) and an Olympus 13.5 megapixel camera (DP-70). Images of sections corresponding to +1.70 , $+1.20,+0.20$ and $-0.40 \mathrm{~mm}$ relative to bregma were analyzed using ImageTool software for lesion development. ${ }^{32}$ The Cavalieri method was used to calculate remaining cortical volume. ${ }^{33}$ The number of sections and the section thickness $(40 \mu \mathrm{m})$ was multiplied by the mean area of cortex for the ipsilateral and contralateral hemisphere and percent remaining cortex were calculated using the equation:

$$
(1-(\text { ipsi/contra }) \times 100) \cdot{ }^{24,34}
$$

Data analysis. Analysis of variance (ANOVA) tests were performed where appropriate for all behavioral and cognitive measures using procedures for general linear models (SPSS 15.0) with options for repeated measures. Between group factors were group ( 4 hr-NAM, 8 hr-NAM, 24 hr-NAM, Saline and Sham). The within group factor was day of testing. Huynh-Feldt probabilities (HFP) and Tukey's HSD test (HSD) were used to correct for Type-1 error associated with repeated measures and post hoc means comparisons. Post-hoc analysis following a significant interaction effect was performed with planned comparison t-tests. A significance level of $\mathrm{p}<0.05$ was used for all statistical analyses. Three rats were removed from the study because of morbidity or mortality and were not replaced. The final group assignments were $4 \mathrm{hr}-\mathrm{NAM}(\mathrm{n}=7), 8 \mathrm{hr}-\mathrm{NAM}(\mathrm{n}=8), 24$ hr-NAM $(\mathrm{n}=8)$, Saline $(\mathrm{n}=8)$ and Sham $(\mathrm{n}=6)$.

\section{Acknowledgements}

The authors would like to thank Jordan Yearwood for his assistance on this project. Research supported by NINDS (NS045647-03) to M.R.H.

\section{References}

1. Narayan RK, MichelME, Ansell B, BaethmannA, BiegonA, BrackenMB, BullockMR, ChoiSC, Clifton GL, Contant CF, Coplin WM, Dietrich WD, Ghajar J, Grady SM, Grossman RG, Hall ED, Heetderks W, Hovda DA, Jallo J, Katz RL, Knoller N, Kochanek PM, Maas AI, Majde J, Marion DW, Marmarou A, Marshall LF, McIntosh TK, Miller E, Mohberg N, Muizelaar JP, Pitts LH, Quinn P, Riesenfeld G, Robertson CS, Strauss KI, Teasdale G, Temkin N, Tuma R, Wade C, Walker MD, Weinrich M, Whyte J, Wilberger J, Young AB, Yurkewicz L. Clinical trials in head injury. J Neurotrauma 2002; 19:503-57.

2. Thurman DJ, Alverson CA, Dunn KA, Guerrero J, Sniezek JE. Traumatic brain injury in the United States: a public health perspective. J Head Trauma Rehabil 1999; 14:602-15.

3. Thurman DJ, Guerrero J. Trends in hospitalization associated with traumatic brain injury. JAMA 1999; 282:954-7.

4. Sahuquillo J, Poca MA, Amoros S. Current aspects of pathophysiology and cell dysfunction after severe head injury. Curr Pharm Des 2001; 7:1475-503.

5. Dickinson K, Bunn F, Wentz R, Edwards P, Roberts I. Size and quality of randomised controlled trials in head injury: review of published studies. BMJ (Clinical research ed) 2000; 320:1308-11.

6. Choi DW. Ionic dependence of glutamate neurotoxicity. J Neurosci 1987; 7:369-79.

7. Rothman S. Synaptic release of excitatory amino acid neurotransmitter mediates anoxic neuronal death. J Neurosci 1984; 4:1884-91.

8. Maiese K, Chong ZZ. Nicotinamide: necessary nutrient emerges as a novel cytoprotectant for the brain. Trends Pharmacol Sci 2003; 24:228-32.

9. Strauss KI, Barbe MF, Marshall RM, Raghupathi R, Mehta S, Narayan RK. Prolonged cyclooxygenase- 2 induction in neurons and glia following traumatic brain injury in the rat. J Neurotrauma 2000; 17:695-711.

10. Virag L, Szabo C. The therapeutic potential of poly(ADP-ribose) polymerase inhibitors. Pharmacol Rev 2002; 54:375-429.

11. Sadanaga-Akiyoshi F, Yao H, Tanuma S, Nakahara T, Hong JS, Ibayashi S, Uchimura H, Fujishima M. Nicotinamide attenuates focal ischemic brain injury in rats: with special reference to changes in nicotinamide and $\mathrm{NAD}^{+}$levels in ischemic core and penumbra. Neurochem Res 2003; 28:1227-34.

12. Tam D, Tam M, Maynard KI. Nicotinamide modulates energy utilization and improves functional recovery from ischemia in the in vitro rabbit retina. Ann N Y Acad Sci 2005; 1053:258-68.

13. Yang J, Klaidman LK, Adams JD. Medicinal chemistry of nicotinamide in the treatment of ischemia and reperfusion. Mini Rev Med Chem 2002; 2:125-34.

14. Horsman MR, Hayer M, Honess DJ, Dennis IF, Overgaard J. Nicotinamide pharmacokinetics in humans and mice: a comparative assessment and the implications for radiotherapy. Radiotherapy and oncology: journal of the European Society for Therapeutic Radiology and Oncology 1993; 27:131-9.

15. Yang J, Klaidman LK, Nalbandian A, Oliver J, Chang ML, Chan PH, Adams JD Jr. The effects of nicotinamide on energy metabolism following transient focal cerebral ischemia in Wistar rats. Neurosci Lett 2002; 333:91-4.

16. Ayoub IA, Lee EJ, Ogilvy C, Flint Beal M, Maynard KI. Nicotinamide reduces infarction up to two hours after the onset of permanent focal cerebral ischemia in wistar rats. Neurosci Lett 1999; 259:21-4.

17. Sakakibara Y, Mitha AP, Ogilvy C, Maynard KI. Post-treatment with nicotinamide (vita$\min B_{3}$ ) reduces the infarct volume following permanent focal cerebral ischemia in female sprague-dawley and wistar rats. Neurosci Lett 2000; 281:111-4.

18. Ayoub IA, Maynard KI. Therapeutic window for nicotinamide following transient focal cerebral ischemia. Neuroreport 2002; 11:213-6. 
19. Hoane MR, Akstulewicz SL, Toppen J. Treatment with vitamin $B_{3}$ improves functional recovery and reduces GFAP expression following traumatic brain injury in the rat. J Neurotrauma 2003; 20:1189-98.

20. Hoane MR, Gilbert DR, Holland MA, Pierce JL. Nicotinamide reduces acute cortical neuronal death and edema in the traumatically injured brain. Neurosci Lett 2006; 35-9.

21. Hoane MR, Kaplan SA, Ellis AL. The effects of nicotinamide on apoptosis and blood-brain barrier breakdown following traumatic brain injury. Brain Res 2006; 1125:185-93.

22. Hoane MR, Tan AA, Pierce JL, Anderson GD, Smith DC. Nicotinamide treatment reduces behavioral impairments and provides cortical protection after fluid percussion injury in the rat. J Neurotrauma 2006; 23:1535-48.

23. Holland MA, Tan AA, Smith DC, Hoane MR. Nicotinamide treatment provides acute neuroprotection and GFAP regulation following fluid percussion injury. J Neurotrauma 2008; 25:140-52.

24. Hoane MR, Pierce JL, Holland MA, Anderson GD. Nicotinamide treatment induces behavioral recovery when administered up to 4 hours following cortical contusion injury in the rat. Neuroscience 2008; in press.

25. Glenn TC, Kelly DF, Boscardin WJ, McArthur DL, Vespa P, Oertel M, Hovda DA, Bergsneider M, Hillered L, Martin NA. Energy dysfunction as a predictor of outcome after moderate or severe head injury: indices of oxygen, glucose and lactate metabolism. Journal of cerebral blood flow and metabolism: official journal of the International Society of Cerebral Blood Flow and Metabolism 2003; 23:1239-50.

26. Tavazzi B, Signoretti S, Lazzarino G, Amorini AM, Delfini R, Cimatti M, Marmarou A, Vagnozzi R. Cerebral oxidative stress and depression of energy metabolism correlate with severity of diffuse brain injury in rats. Neurosurgery 2005; 56:582-9.

27. Lindner MD, Plone MA, Cain CK, Frydel BR, Francis JM, Emerich DF, Sutton RL. Dissociable long-term cognitive deficits after frontal versus sensorimotor cortical contusions. J Neurotrauma 1998; 15:199-216.

28. Barth TM, Jones TA, Schallert T. Functional subdivisions of the rat somatic sensorimotor cortex. Behav Brain Res 1990; 39:73-95.

29. Hoane M, Wolyniak J, Akstulewicz S. Administration of riboflavin improves behavioral outcome and reduces edema formation and GFAP expression following traumatic brain injury. J Neurotrauma 2005:1112-22.

30. Schallert T, Woodlee MT. Orienting and Placing. In: Whishaw IQ, Kolb B, eds. The Behavior of the Laboratory Rat. New York: Oxford University Press 2005; 129-40.

31. Hoane MR, Raad C, Barth TM. Non-competitive NMDA antagonists and anti-oxidant drugs reduce striatal atrophy and facilitate recovery of function following lesions of the rat cortex. Restor Neurol Neurosci 1997; 11:71-82.

32. Paxinos G, Watson C. The rat brain in stereotaxic coordinates. New York: Elsevier 2005

33. Coggeshall RE. A consideration of neural counting methods. TINS 1992; 15:9-13.

34. Hoane MR, Pierce JL, Holland MA, Birky ND, Dang T, Vitek MP, McKenna SE. The novel apolipoprotein E-based peptide COG1410 improves sensorimotor performance and reduces injury magnitude following cortical contusion injury. J Neurotrauma 2007; 24:1108-18. 


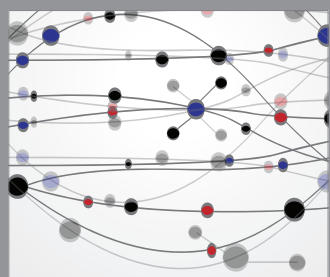

The Scientific World Journal
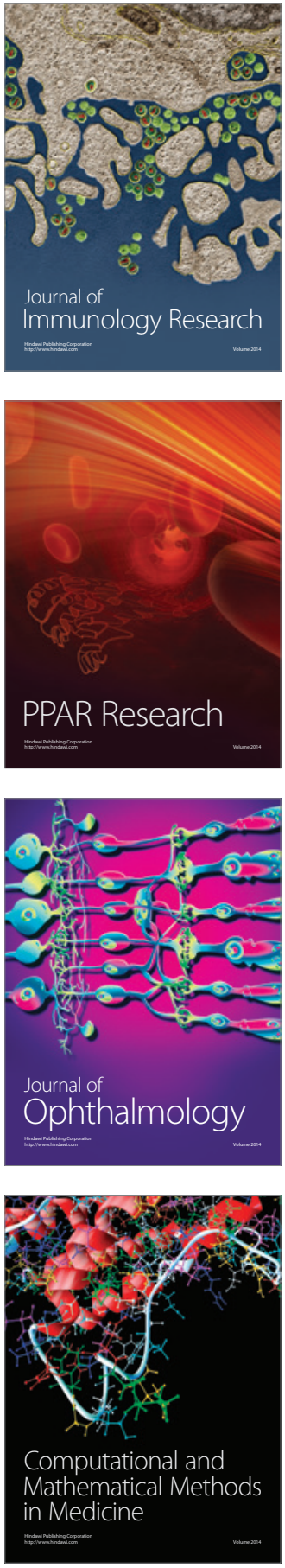

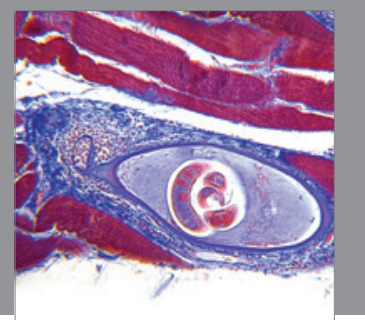

Gastroenterology

Research and Practice
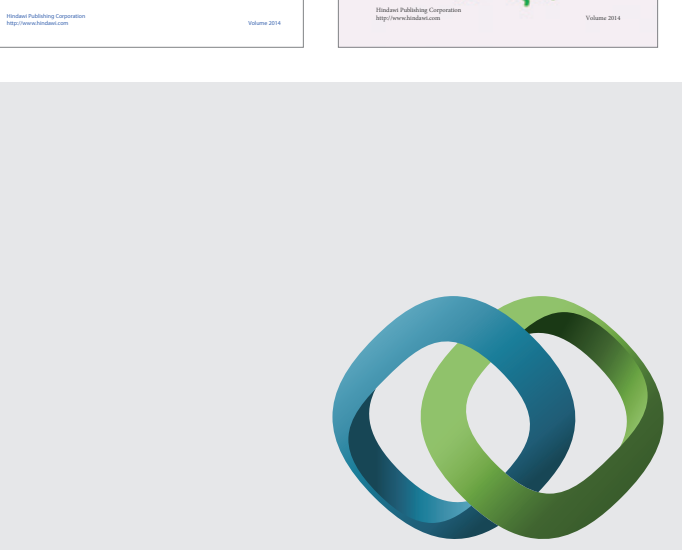

\section{Hindawi}

Submit your manuscripts at

http://www.hindawi.com
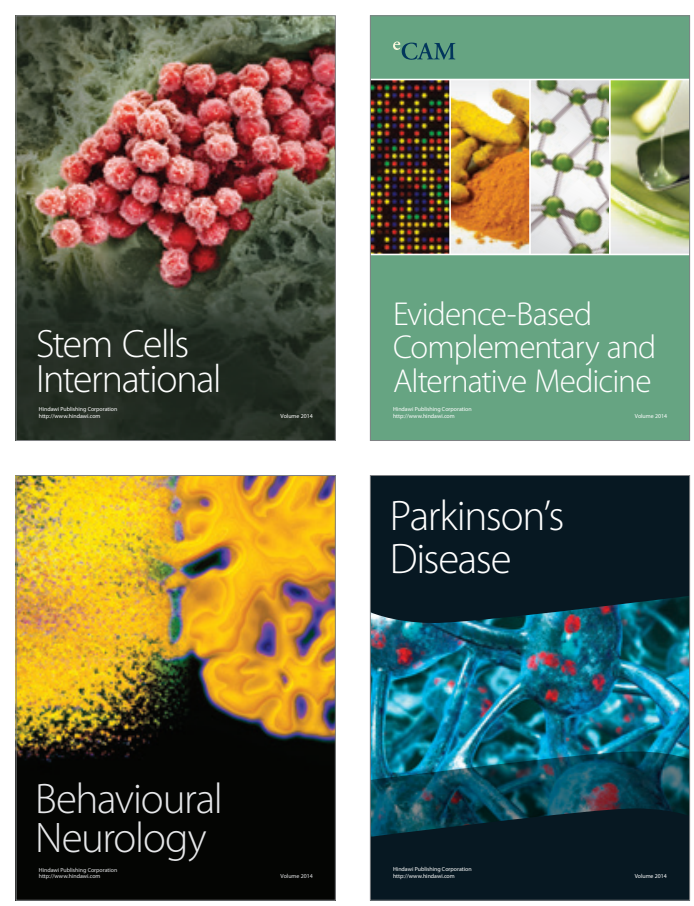

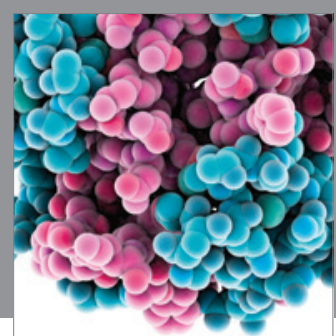

Journal of
Diabetes Research

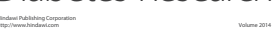

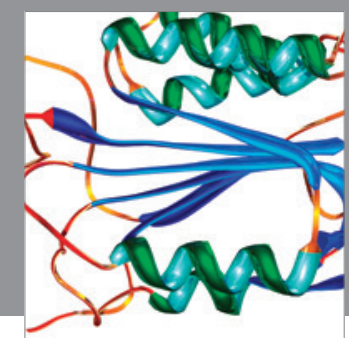

Disease Markers
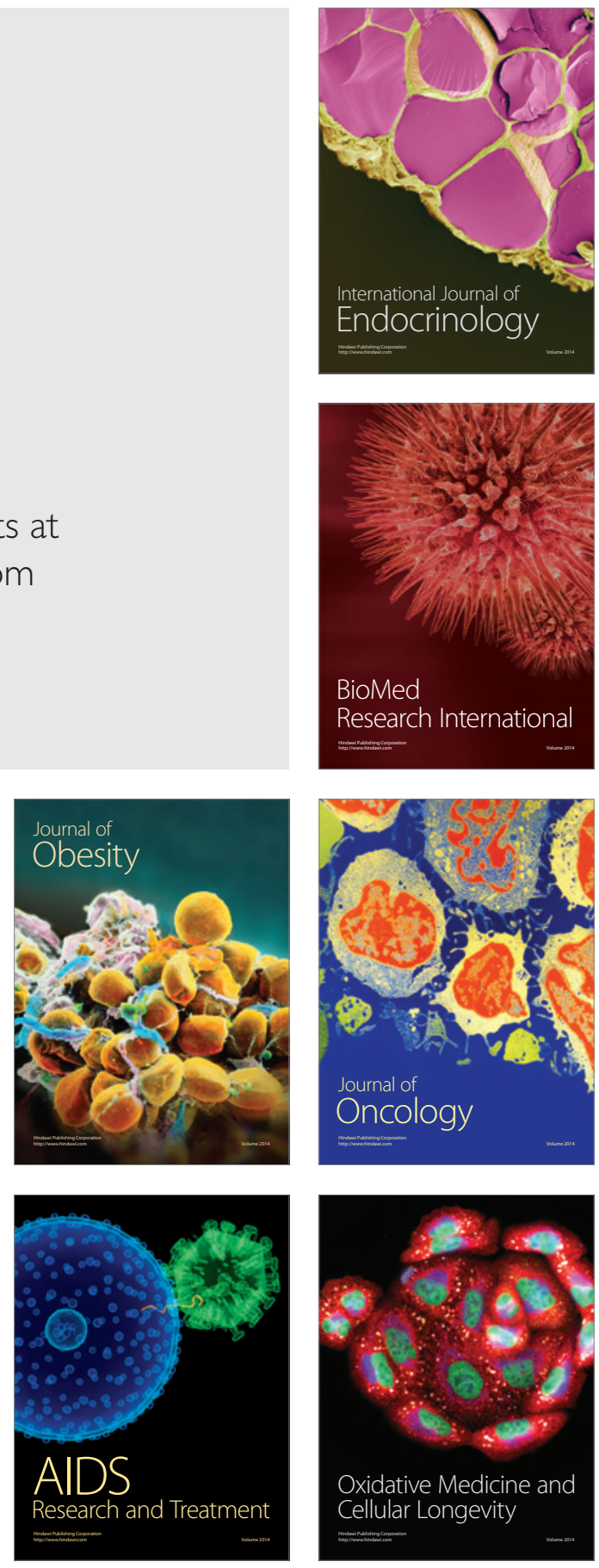